\title{
Situação endêmica da hanseníase em uma cidade do interior do Rio Grande do Sul
}

\author{
Endemic situation of leprosy in a city of the countryside of Rio Grande do Sul
}

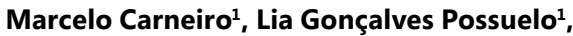 \\ Andréia Rosane Moura Valim ${ }^{1}$, Luciano Nunes Duro ${ }^{1}$ \\ ${ }^{1}$ Professores do curso de medicina (Unisc), Santa Cruz do Sul - RS - Brasil
}

carneiromarcelo@yahoo.com.br

\section{Resumo}

Justificativa e Objetivos: $O$ controle da hanseníase é uma prioridade de saúde pública, no entanto, as baixas prevalências encontradas no Rio Grande do Sul favorecem o subdiagnóstico. O objetivo foi avaliar os pacientes com hanseníase e seus comunicantes, no período de 1995-2005, na cidade de Santa Cruz do Sul, RS, a fim de realizar uma busca ativa de novos casos. Método: Foi um estudo retrospectivo com análise dos 38 prontuários e a situação de seus 67 contatos do período analisado. Resultados: A média de idade foi de 50,5 anos, sem predomínio de gênero. A maioria

\section{Abstract}

Background and Objectives: The control of leprosy is a public health priority, however, the low prevalence in Rio Grande do Sul favoring underdiagnosis. The objective was to evaluate patients with leprosy during 1995 to 2005 in the city of Santa Cruz do Sul, RS. Results: We performed a retrospective analysis of 38 medical records and assessed the situation of 67 contacts. The mean age was 50.5 years, without gender dominance. The majority (87.2\%)

\section{Introdução}

A hanseníase no Brasil, apesar da redução da prevalência de 4,93/10.000 (1998) para 1,48/10.000 (2005), continua sendo uma doença negligenciada e um problema de saúde pública. Dentre os estados brasileiros, o Rio Grande do Sul (RS) apresenta
(87,2\%) eram agricultores caucasóides com baixo nível de escolaridade (74\%). Houve predominância de casos multibacilares (71,1\%), forma clínica virchowiana (52\%) e incapacidade funcional verificada em $36,8 \%$. Apenas um contato com hanseníase foi identificado. Conclusão: $O$ monitoramento de uma área de baixa prevalência deverá priorizar o diagnóstico precoce, ou seja, hanseníase paucibacilar, favorecendo a prevenção de incapacidades físicas. Palavras chaves: Controle de doenças transmissíveis, Hanseníase, Lepra, Mycobacterium leprae.

were Caucasian farmers with low education (74\%). Multibacillary cases predominated (71.1\%), clinical form virchowiana (52\%) and functional incapacity was found in $36.8 \%$. Only one contact with leprosy was identified. Conclusion: The control of a low prevalence area should prioritize early detection, facilitating the prevention of disabilities. Keywords: Communicable disease control, Hansen's disease, Leprosy, Mycobacterium leprae.

a menor taxa de prevalência $(0,53 / 10.000)^{1}$. O objetivo deste estudo foi analisar a situação dos casos registrados entre 1995 e 2005, no município de Santa Cruz do Sul, para subsidiar estratégias de controle da doença. 


\section{Métodos}

Após aprovação pela Comissão de Ética da Universidade Santa Cruz do Sul, RS, sob protocolo no 2634/10 (CAAE 0137.0.109.000-10), realizou-se este estudo observacional, clínico-epidemiológico, do tipo transversal, no Ambulatório de Micobacterioses, da Secretaria Municipal de Saúde, no período de 1995 até 2005, na cidade de Santa Cruz do Sul, RS, localizada na região do Vale do Rio Pardo, a $155 \mathrm{~km}$ da capital Porto Alegre. Apresenta, aproximadamente, 120.000 habitantes com uma população predominantemente $(87 \%)$ urbana com renda per capita de U\$\$12.834,02.

O estudo foi conduzido dentro dos padrões exigidos pela Declaração de Helsinque.

A primeira fase do estudo foi retrospectiva, e contou com as informações dos prontuários médicos e de fichas do Sistema de Informações de Agravos de Notificação (SINAN) de pacientes inscritos, e de seus comunicantes intradomiciliares. $\mathrm{Na}$ segunda fase foram realizadas entrevistas (por telefone e pessoalmente), além de exame físico dos indivíduos tratados e seus contatos. Foram considerados contatos intradomiciliares aqueles que residem ou residiram no mesmo domicílio do doente no período de diagnóstico. Os dados epidemiológicos referentes às condições socioeconômicas, demográficas, ambientais e clínicas, foram colhidos utilizando um questionário epidemiológico estruturado para o estudo. Os dados foram armazenados e analisados, utilizando-se o programa SPSS ver. 13.0 (SPSS, Chicago, IL). Os valores foram expressos como médias ou como números e porcentagens. A comparação das variáveis categóricas entre os grupos foi realizada utilizando o teste $\chi^{2}$.

\section{Resultados}

Foram analisados 38 prontuários de pacientes com diagnóstico de hanseníase, pós-alta da poliquimioterapia (PQT) no período estudado.
A média de idade dos pacientes no momento do diagnóstico foi de 50,5 anos, variando de 20 a 75 anos. Vinte eram mulheres (52,6\%). A zona urbana da cidade era o local de residência de $97,4 \%$, sendo $87,2 \%$ da cor branca. Dezessete pacientes (44\%) relataram como principal ocupação a agricultura (plantação e colheita de tabaco). Do total de pacientes analisados, 22 (58\%) concluíram apenas o ensino fundamental e 6 (16\%) eram analfabetos.

A história de sinais/sintomas da doença variou, em média de 3,8 anos (mínimo de um e máximo de 25 anos) antes do diagnóstico. A forma clínica mais frequente de acordo com a Classificação de Madri, foi a virchowiana, em 20 (52,6\%) casos $(p=0,03)$ e baseada na classificação operacional da Organização Mundial de Saúde, 27 (71,1\%) casos foram incluídos no grupo multibacilar (Tabela 1).

Com relação à baciloscopia, 14 (36,8\%) doentes apresentaram baciloscopia positiva, 15 (39,5\%) baciloscopia negativa e em 9 (23,7\%) não foi realizada a baciloscopia.

As lesões mais prevalentes, no diagnóstico, foram manchas avermelhadas e anestésicas esparsas nos membros e tronco em 28 pacientes $(73,7 \%)$. Úlceras estavam presentes em $21 \%$ dos casos, tanto nas mãos quanto nos pés. Na avaliação do grau de incapacidade física, observou-se que 15 (40\%) apresentavam algum grau de incapacidade, sendo $26,6 \%$ com grau I e $73,4 \%$ com grau II. Na avaliação pós-alta as deformidades mais observadas foram úlceras plantares (13\%), artropatia de Charcot $(9,0 \%)$ e flexão fixa ("garra") dos dedos das mãos (6\%).

Um total de $34(89,5 \%)$ doentes, não havia realizado tratamento anterior. Quatro $(10,5 \%)$ doentes estavam em retratamento, em média, 11 anos após o primeiro diagnóstico da doença. $\mathrm{O}$ tratamento anterior utilizado por estes doentes foi a monoterapia sulfônica. Todos os doentes incluídos no estudo receberam alta por cura. Independente da alta da PQT, os pacientes continuaram assistidos, por profissionais da saúde, na unidade básica de saúde, devido a possíveis reações hansênicas 
e sequelas neurológicas. As reações hansênicas ocorreram em $7(18,4 \%)$ pacientes após a alta, sendo que $3(42,8 \%)$ pacientes apresentaram reação reversa (tipo 1 ) e $4(57,1 \%)$ desenvolveram eritema nodoso hansênico (tipo 2). A neurite aguda foi verificada em $30,2 \%$ de todos os casos das reações do tipo 1 e 2 .

$\mathrm{Na}$ análise das fichas do SINAN, a média de comunicantes foi de três pessoas/domicílio para cada doente e nas entrevistas pós-tratamento os contatos diminuíram para duas pessoas em cada domicílio. Foram avaliados 67 contatos intradomiciliares dos doentes tratados. A maioria dos doentes tinha como contato os cônjuges $(72,2 \%)$ e os filhos (40,3\%). Observou-se que apenas um $(1,5 \%)$ contato intradomiciliar adoeceu. A vacina $B C G$ foi oferecida para todos os contatos com $100 \%$ de adesão.

\section{Discussão}

Na análise clínica e epidemiológica dos doentes de hanseníase observou-se a predominância de população urbana e caucasóide, característica da região que é de descendentes imigrantes alemães. Não se observou predominância de gênero $(p=0,75)$ como o descrito por Gomes e col. ${ }^{3}$. A doença acometeu pessoas em idade economicamente ativa, fato que sobrecarrega e onera o sistema de saúde pública e previdenciário do Estado. As orientações preventivas e terapêuticas deverão respeitar e considerar a individualidade e o contexto familiar, a fim de minimizar as conseqüências da estigmatização na vida social e na cadeia produtiva6.

Observou-se uma predominância de casos multibacilares $(71,1 \%)$ sob a forma virchowiana $(52,7 \%)$. Estes resultados estão de acordo com aqueles apresentados por Sanches e col. ${ }^{4}$ que avaliaram 222 pacientes com hanseníase, no Estado do Paraná, dos quais $63,0 \%$ eram multibacilares, tendo o grupo virchowiano, com maior representatividade (81,3\%). Ignotti e col. ${ }^{4}$ concluíram que a proporção de pacientes com lesão única de pele pode ser utilizada como indicador na avaliação do progresso da eliminação da hanseníase em áreas hiperendêmicas. $O$ alto percentual das formas multibacilares demonstra atraso no diagnóstico, permitindo questionar a falta de detecção de casos nas formas iniciais pela rede básica de saúde, reforçando a importância da capacitação para as equipes de saúde.

Parte importante da população estudada (40\%) já apresentava algum tipo de alteração neurológica no diagnostico da doença. Esse fato se torna relevante, pois implicaria no desenvolvimento de ações educativas e preventivas das complicações de incapacidades físicas, no dia da primeira consulta médica.

A demanda espontânea, de novos casos, foi responsável por $50 \%$ dos diagnósticos feitos pelo serviço, demonstrando a importância dos meios de comunicação e do esclarecimento sobre a doença e suas incapacidades. Estes dados evidenciam que a procura de auxílio médico foi nas formas mais graves e contagiosas da doença, contribuindo para a manutenção do ciclo de transmissão. Em $21 \%$ dos casos foi verificada a presença de úlceras, tanto nas mãos quanto nos pés. Este tipo de manifestação clínica pode servir de indicativo para procura de novos casos, na população de pessoas com mal perfurante plantar sem causa definida.

As reações cutâneas pós-tratamento ocorreram em $18,4 \%$ dos pacientes após a alta. Não foram observadas diferenças significativas $(p=0,74)$ com relação ao tipo de reação. A identificação precoce das manifestações relacionadas às reações hansênicas previnem o desenvolvimento de neurite aguda o que pode gerar incapacidade física do membro afetado e possível sequela irreversível.

A baixa freqüência de casos (1,5\%) entre os comunicantes de doentes com hanseníase é esperado, apesar de ser um dos fatores de risco para adquirir a doença².

O controle epidemiológico de uma área de baixa prevalência deverá prever o controle periódico dos comunicantes, pois os casos analisados foram de diagnóstico tardio, isto é, subdiagnóstico, especialmente, para nas formas clínicas paucibacilares. 
Tabela 01 - Distribuição dos casos de hanseníase em Santa Cruz do Sul, RS, 1995-2005.

\begin{tabular}{|c|c|c|}
\hline CARACTERÍSTICAS & $\begin{array}{l}\text { Total } \\
\text { n }(\%)\end{array}$ & $\mathbf{P}$ \\
\hline Sexo & & 0,75 \\
\hline Feminino & $20(52,7)$ & \\
\hline Masculino & $18(47,3)$ & \\
\hline \multicolumn{3}{|l|}{ Faixa etária* } \\
\hline 15- 24 & $3(7,9)$ & \\
\hline $25-34$ & $2(5,3)$ & \\
\hline $35-44$ & $9(23,7)$ & \\
\hline $45-54$ & $13(34,2)$ & \\
\hline $55-65$ & $1(2,6)$ & \\
\hline$>65$ & $10(26,3)$ & \\
\hline Classificação operacional & & 0,03 \\
\hline Multibacilar & $27(71,1)$ & \\
\hline Paucibacilar & $11(28,9)$ & \\
\hline Formas clínicas & & 0,03 \\
\hline Tuberculoide & $7(18,4)$ & \\
\hline Dimorfa & $7(18,4)$ & \\
\hline Virchowiana & $20(52,7)$ & \\
\hline Indeterminada & $4(10,5)$ & \\
\hline Baciloscopia & & 0,66 \\
\hline Positiva & $14(36,8)$ & \\
\hline Negativa & $15(39,5)$ & \\
\hline Não realizada & $9(23,7)$ & \\
\hline Grau de incapacidade** & & 0,28 \\
\hline Grau I & $4(26,6)$ & \\
\hline Grau II & $11(73,4)$ & \\
\hline Reações hansênicas & $7(18,4)$ & 0,74 \\
\hline Reação reversa (tipo I) & $3(42,8)$ & \\
\hline ENH (tipo II) & $4(57,2)$ & \\
\hline
\end{tabular}

\section{REFEFÊNCIAS}

1. Brasil, Ministério da Saúde. Secretaria de Vigilância Sanitária. Departamento de Vigilância Epidemiológica. Hanseníase no Brasil. $1^{\circ}$ edição. Brasília: Ministério da Saúde, 2009.

2. Castro NMC, Restrepo-Jaramilo S, Ossa MG, Brennan PJ. Infection by Mycobacteriium leprae of household contacts of lepromatous leprosy patients from a post-elimination leprosy region of Colômbia. Mem Inst Oswaldo Cruz. 2005;100(7):703-707.

3. Gomes CCD, Pontes MAA, Gonçalves HS, Penna GO. Perfil clínico-epidemiológico dos pacientes diagnosticados com hanseníase em um centro de referência na região nordeste do Brasil. Anais Bras Dermatol. 2005;80 (Supl 3):283-288.

4 Ignotti E, Bayona M, Alvarez-Garriga C, Andrade VLG, Valente JG. Single lesion as an indicator to monitor the leprosy trend to elimination in hyperendemic areas. Rev Bras Epidemiol. 2007;10(3):421-431.

5. Oliveira MHP, Romanelli G. Os efeitos da hanseníase em homens e mulheres: um estudo de gênero. Cad Saúde Pública. 1998;14(1):51-60.

6. Sanches LAT, Pittner E, Sanches HF, Monteiro MC. Detecção de casos novos de hanseníase no município de Prudentópolis, PR: uma análise de 1998 a 2005. Rev Soc Bras Med Trop. 2007;40(5):541-545. 